\title{
The LIKELIHOOd OF ACHIEVING QUANTIFIED RoAd SAFETy TARgets: A Binary Logistic Regression Model For Possible FACTORS
}

\author{
N.N. SZE (Corresponding Author) \\ Department of Civil and Natural Resources Engineering, University of Canterbury, Christchurch, \\ New Zealand \\ Email: tony.sze@canterbury.ac.nz, Tel. +64 3 364-2238, Fax.+64 33642758 \\ S.C. WONG \\ Department of Civil Engineering, The University of Hong Kong, Pokfulam Road, \\ Hong Kong, China
}

E-mail: hhecwsc@ hku.hk, Tel: +852 2859-1964, Fax: +852 2559-5337

14

C.Y. LEE

Department of Civil Engineering, The University of Hong Kong, Pokfulam Road, Hong Kong, China Email: cycyemilylee@ hotmail.com, Tel: +852 2859-8024, Fax: +852 2559-5337

\begin{abstract}
In past several decades, many countries have set quantified road safety targets to motivate transport authorities to develop systematic road safety strategies and measures and facilitate the achievement of continuous road safety improvement. Studies have been conducted to evaluate the association between the setting of quantified road safety targets and road fatality reduction, in both the short and long run, by comparing road fatalities before and after the implementation of a quantified road safety target. However, not much work has been done to evaluate whether the quantified road safety targets are actually achieved. In this study, we used a binary logistic regression model to examine the factors - including vehicle ownership, fatality rate, and national income, in addition to level of ambition and duration of target - that contribute to a target's success. We analyzed 55 quantified road safety targets set by 29 countries from 1981 to 2009, and the results indicate that targets that are in progress and with lower level of ambitions had a
\end{abstract}


1 higher likelihood of eventually being achieved. Moreover, possible interaction effects on the association between level of ambition and the likelihood of success are also revealed.

4 Keywords: Road safety target; Road fatality; Binary logistic regression; Level of Ambition; Interaction Effect

\section{INTRODUCTION}

There has been a global reduction in road fatalities over the past three decades due to the adoption of various road safety programs and policies, especially in European Union (EU) Member States. Organizations such as the World Health Organization (WHO), the Organization for Economic Co-operation and Development (OECD), the European Transport Safety Council (ETSC), and International Transport Research Documentation (ITRD) are committed to establishing systematic road safety management systems, setting quantified road safety targets, and motivating timely action plans to achieve continuous and considerable road fatality reductions (Elvik, 1993; OECD Scientific Expert Group, 1994, 2008; Wegman et al., 2008; ETSC, 2011).

Quantitative road safety targets for fatality reductions must be ambitious and yet achievable (ETSC, 2003). Studies have been conducted to estimate the degree to which a target has been realized by comparing the road fatalities before and after the deployment of the respective target using a treatment-comparison group approach (Elvik, 2001; Allsop et al., 2011). Compared with countries lacking targets, target deployment was found to correlate with significant road fatality reductions, both in the short and long run. More importantly, a favorable effect on the time-series trend of road fatalities was observed during the period the target was in effect (Wong et al., 2006; Wong and Sze, 2010).

Although the sustainable favorable effects on fatality reduction have been realized, it is essential to determine appropriate targets that can lead to successful safety improvements. Based on information about current and future road safety performance forecasts, implementation plans for different road safety policies and measures, and the predicted effects of individual road safety actions, it is possible to estimate the likelihood of achieving a road safety target. Therefore, realistic quantified road safety targets can be set by referring to the existence of legislative and 
1 engineering interventions and the implementation of such interventions, controlling for the timeseries trend of road safety level (Kweon, 2010). In addition, inputs from safety experts on the likelihood of and reasons for target achievement are essential. Attempts have been made to set appropriate targets for individual EU Member States to achieve optimal road fatality reduction throughout the EU as a whole. For instance, appropriate quantified road safety targets were set based on historical road safety trends, predicted future potentials, the capability to implement road safety measures, the achievement of preceding targets, interest and focus, and the economic status of individual EU Member States (Wittenberg et al., 2013).

Stimulating effective road safety management systems with policy documents, action plans, and guidelines is essential to the success of road safety targets. Attempts have been made to categorize individual EU Member States based on various road safety performance indicators with respect to different exposure measures, including population, vehicle fleet, and vehicle kilometric. In particular, the effectiveness of the road safety management systems of individual Member States has been benchmarked against better performing Member States in terms of policy making, action planning, and setting road safety targets in respective categories (Shen et al., 2012). Various safety performance indicators related to road infrastructure, vehicle technology, and road user behavior can be generated and a composite safety performance indicator adopted as a management tool for setting appropriate road safety targets in the development of appropriate road safety strategies and countermeasures (Tingvall et al., 2010). A better understanding of optimal target setting and its relationship with existing road safety management systems as it pertains to the predicted effect of time-series trends in road safety is expected to noticeably enhance road fatality reduction and sustained safety performance.

Nevertheless, it is desirable to evaluate the level of achievement in countries setting quantified road safety targets by comparing the targeted and actual reductions in road fatalities. In this study, we evaluate the performance of 55 quantified road safety targets set by 29 countries using a cluster analysis to categorize the targets into groups with similar characteristics, such as fatality rate, vehicle ownership, national income, time of target setting, and level of ambition. A binary logit regression is applied to assess the success of the targets and the relationships between the likelihood of achievement and possible factors including fatality rate, vehicle ownership, level of ambition, target duration, and whether a target is completed or not. Comparing the targeted and 
1 revealed reductions in fatalities in countries with quantified road safety targets should provide insights that could be valuable to decision makers in setting appropriate targets.

4 The remainder of the paper is structured as follows. The data on quantified road safety targets and road fatalities used in this study are described in Section 2. Section 3 details the application of the cluster analysis in categorizing the identified quantified road safety targets. The results of the binary logistic regression of the likelihood of target achievement and possible influencing factors are presented in Section 4. Section 5 discusses the implications of our results and provides recommendations for planning road safety strategies and programs. We conclude the study with suggestions for future research in Section 6.

\section{DATA}

The focus of this study is the achievement of road fatality reductions in target years through quantified road safety targets. The details of established quantified road safety targets are the fundamentals of this study. Information on 55 targets in 29 selected countries, including the base year, target period, and target road fatality reduction, for the period 1981-2009 are obtained from key publications issued by the Institute of Transport Economics in Norway and the OECD Scientific Expert Group (Elvik, 2001; OECD Scientific Expert Group, 2012, 2014), in addition to the national study reports and technical papers listed in the References. The details of the quantified road safety targets under investigation are presented in Table 1 . The fatality data for the 1981-2012 period are extracted from the International Road Federation (IRF) World Road Statistics (International Road Federation, 2013).

[Insert Table 1 here]

As shown in Table 1, of the 55 targets, 7 were established in the 1980s, 21 were established in the 1990s, and almost one half $(27,49 \%)$ were established in the 2000s. Summary statistics of the 55 targets under investigation are presented in Table 2.

[Insert Table 2 here] 
1 As shown in Table 2, the target period ranges from 2 to 17 years, with an average of 9 years. In 2 2012, 46 (84\%) targets were completed and 9 (16\%) were in progress respectively. Variation in 3 the levels of ambition is substantial. The targeted percentage fatality reduction ranges from $0 \%$ to $56 \%$, with an average of $34 \%$, compared with the number of road fatalities in the base year. Given the information on the target period and targeted fatality reduction, the targeted annual reduction rate (ARR) can be deduced by,

$$
A R R=1-T^{1 / n}
$$

(Equation 1)

where $T$ is the targeted percentage fatality reduction and $n$ is the target period. $A R R$ is a proxy for the target's level of ambition. As also shown in Table 2, the mean annual average reduction rate is $5 \%$.

As the fatality data up to 2012 are adopted, the number of fatalities in the corresponding target year can be used to assess target completion. For targets in progress, the number of fatalities in 2012 is used to benchmark the achievement of the corresponding targets. To ensure that the effect estimates are not double counted, for two or more targets that are set by the same country in the same year and with target years being after 2012, we would select the one that have the earliest target year. Besides, as it takes time for any road safety initiatives to be in effect upon the target setting, targets that are established at a later time after 2009 are not selected.

For the selected targets, information on possible influencing factors, including fatality rate, vehicle ownership, national income, year of implementation, target period, target completion, and targeted fatality reduction rate, is extracted. Road fatality per 100,000 inhabitants has been a common road safety risk indicator in public health sector. Information on population is readily available in most countries, as compared to other exposure measures like vehicle kilometric. Fatality rate using population as denominator is particular useful to compare the fatality risks in countries with similar levels of motorization. In 2012, road fatalities per 100,000 inhabitants have been the lowest in five European countries including Great Britain, Denmark, Iceland, Norway, and Sweden (OECD, 2014). Number of vehicle registered per 1000 inhabitants has been a possible indicator for comparing the level of mobility and road infrastructure development across countries, when information on road asset (in terms of total length of highway link) and amount of travel (in terms of vehicle-kilometres) is not always readily available in all countries. GDP per capita is a good indicator for comparing the level of economic 
1 development across different countries. Besides, economic cost of road crash to a country is 2 often presented in terms of proportion of national income. A road safety target could be established before the end of preceding target in one country. It could be because the earlier target was not reached despite of the considerable fatality reduction. This might be attributed to the changes in unforeseen economic growth, increase in vehicle number, and changes in traffic volume and traffic pattern. A review of road safety development and change in strategic priorities could be implemented when a new road safety target was to be established before the end of a preceding target.

\section{CLUSTERS OF QUANTIFIED ROAD SAFETY TARGETS}

Before we measure the associations between target achievement and possible influencing factors, we conduct a cluster analysis to categorize groups of targets with similar characteristics (Depaire et al., 2008; Wong and Chung, 2008; Anderson, 2009). Cluster analysis is an exploratory data analysis technique that identifies interesting patterns of similarity among individuals in a dataset to stimulate further analyses based on an understanding of meaningful patterns of similarity and dissimilarity across individuals.

We apply the agglomerative hierarchical classification approach using the Wald method to establish clusters of quantified road safety targets. In particular, the degree of similarity between individuals is determined by the Euclidean distance. To this end, the possible factors are fatality rate (per 100,000 inhabitants), vehicle ownership (per 1,000 inhabitants), national income (GDP per capita), target duration, targeted annual reduction rate, and base year of target setting. The cluster analysis is performed on R3.0 (R Core Team, 2013). Figure 1 illustrates the dendrogram for the merging progress of individual targets, and the final results of the cluster analysis.

[Insert Figure 1 here]

The dendrogram obtained from the cluster analysis identifies three clusters of quantified road safety targets: (i) targets set up by countries that have low national income (mean $=22,000$ USD per capita) and high fatality rate (mean $=10.1$ per 100,000 inhabitants) (number of targets $=11$, percentage of total $=20.0 \%$ ) (ii) high national income $(45,000$ USD per capita) and low- 
1 medium fatality rate (4.6 per 100,000 inhabitants) (19, 34.5\%); and (iii) medium national income 2 (35,000 USD per capita) and low-medium fatality rate (4.9 per 100,000 inhabitants) $(25,45.5 \%)$.

4 To assess the independence of the clusters, $\mathrm{F}$ tests are conducted to describe the differences across clusters. The results of the F-tests indicate that the differences in the values of the variables of interest, that is, national income $(F=122.5)$ and fatality rate $(16.7)$, across clusters are both significant at the $1 \%$ level.

\section{FACTORS INFLUENCING TARGET SUCCESS}

We aim to evaluate the extent to which setting quantified road safety targets achieves the expected fatality reductions, and to identify the possible factors that contribute to such success. A target is regarded as being achieved if the observed number of road fatalities in the target year (or year 2012) is lower than that of the expected number of road fatalities for the completed target (or the target in progress). The dichotomous nature of the dependent variable (achievement of target) facilitates the application of binary logistic regression. The associations between target success and possible influencing factors are estimated using the maximum likelihood approach, in which the influence of possible factors on the likelihood of success is reflected in the respective odds ratios, and level of significance of their influences on the likelihood of success is reflected in the respective $p$-level respectively (Hosmer and Lemeshow, 2000). To evaluate the goodness-of-fit of the regression model, Akaike Information Criterion (AIC) of the fitted model is deduced by,

where $L L$ is the log-likelihood value and $k$ is the number of parameters in the fitted model. When comparing prediction performance of models fitted using maximum likelihood approach, the smaller the value of $A I C$, the better the model fit. Moreover, to identify possible collinearity among variables in the model fitted, Variance Inflation Factor (VIF) is estimated by,

$$
V I F=1 /\left(1-R_{j}^{2}\right)
$$

(Equation 3)

where $R_{j}^{2}$ is the coefficient of determination for a regression of variable $j$ on all the other variables. A VIF of 10 and above indicates a multicollinearity problem. Variable with high value of VIF (greater than 10) will therefore be removed. 
2 The possible influencing factors in the proposed prediction model are level of ambition 3 (measured by annual reduction rate, ARR), duration of target, vehicle ownership, national 4 income, fatality rate, and completion of target. As revealed in the cluster analysis, the national 5 income and fatality rate are deterministic to the patterns of the targets set, a cluster's effect as 6 established in the preceding cluster analysis will be incorporated to proxy the effects of 7 economic status and prevailing road safety risk on the success of road safety target. Therefore, 8 factors including national income and fatality rate will not be included in subsequent analyses.

[Insert Table 3 here]

A non-parametric test for independence between potential factors and likelihood of success is conducted, followed by regression analysis of the association measure of likelihood of success. As shown in Table 3, vehicle ownership of 650 per 1,000 inhabitant, target duration of 10 years and annual average reduction rate of $4.5 \%$ are set as the cut-offs for vehicle ownership, target duration, and target's level of ambition respectively. Based on the results of non-parametric test, the vehicle ownership, target's level of ambition, and completion of target are selected as candidate factors at the $15 \%$ level of significance. Because the target duration is of primary interest in this study and long-term targets have a higher likelihood of success (Wong and Sze, 2010), target duration is incorporated into subsequent analyses, regardless of its statistical significance in the preliminary analysis.

The results of the consolidated logistic regression analysis of the association measure of target achievement are presented in Table 4. To evaluate the effects of economic status and prevailing road safety risk, a cluster effect is also incorporated into the proposed logistic regression models.

\section{[Insert Table 4 here]}

Table 4 presents the results of the analysis of the association measure between road safety target success and possible influencing factors. For instances, factors including vehicle ownership, target duration, and target's level of ambition are set either as continuous (Model A) or categorical (Model B). As Table 4 reveals, prediction performance of Model B (AIC of 71.8) is superior to that of Model A (75.4). Besides, values of VIF of the variables included in both 
1 models are all below 2. No evidence could be established for the existence of collinearity among 2 the variables.

4 As Model B of Table 4 shows, targets with annual reduction rate higher than or equal to $4.5 \%$ $5 \quad($ Odds ratio $=0.11,95 \%$ CIs $=[0.03,0.46])$ have a lower likelihood of success, at the $1 \%$ level of 6 significance. Besides, completed targets (Odds ratio $=0.15,95 \% \mathrm{CIs}=[0.02,1.13])$ have a 7 marginally lower likelihood of success, at the $10 \%$ level of significance. As also shown in Table 84 , members of Cluster 1 (targets set by countries of low national income and high fatality rate) and Cluster 3 (medium national income and low-medium fatality rate) might have higher likelihood of success, as compared to that of Cluster 2 (high national income and low-medium fatality rate), but no statistical evidence could be established.

To investigate the possible intervention effect by cluster on the association, the interaction effects between cluster and annual reduction rate and between cluster and target duration are also determined, as revealed in Table 5.

[Insert Table 5 here]

Factors including vehicle ownership, target duration, and target's level of ambition are to set be categorical. As Table 5 reveals, prediction performance of interaction model (AIC of 76.0) is not superior to that of prior model (Model B of Table 4). Yet, values of VIF of the variables included in the model are all below 4. No evidence could be established for the existence of collinearity among the variables.

As Table 5 shows, no evidence could be established for the existence of interaction effects between cluster and target duration and between cluster and target's level of ambition on the likelihood of success. As also shown in Table 5, targets with annual reduction rate higher than or equal to $4.5 \%($ Odds ratio $=0.09,95 \% \mathrm{CIs}=[0.01,1.24])$ and completed targets $($ Odds ratio $=$ $0.13,95 \%$ CIs $=[0.01,1.16])$ have marginally lower likelihood of success, both at the $10 \%$ level of significance.

\section{DISCUSSION}


1 Preceding studies have revealed a strong association between the setting of quantified road safety targets and enhanced road safety performance, as indicated by remarkable fatality reductions in the short-medium term and favorable trends in road safety performance (stronger fatality reduction or weaker fatality growth) in the long term after the setting of targets in OECD countries from the 1970s to the 1990s (Wong et al., 2006; Wong and Sze, 2010). We revisit the issue to diagnose the success of target achievement. In particular, we use a logistic regression approach to identify the factors that may contribute to such success, including level of ambition, target duration, vehicle ownership, economic status and prevailing road safety risk. The results indicate that target's level of ambition is associated with target achievement.

\subsection{Target Clusters}

Cluster analysis is applied to understand the variations in the patterns of road safety target established, and the possible influences on the pattern by the factors including vehicle use, prevailing road safety risk, and economic development. As the results of the cluster analysis indicate, targets set can be stratified into three groups: (i) those that were established by the countries with low national income and high fatality rate (Cluster 1); (ii) high national income and low-medium fatality rate (Cluster 2); and (iii) medium national income and low-medium fatality rate (Cluster 3). The results of the logistic regression suggest that no evidence could be established for noticeable association between cluster and likelihood of success, and interactions by cluster on the association between level of ambition, target duration, and likelihood of success.

Seemingly, targets set by members of Cluster 2 (high national income and low-medium fatality rate) might have a lower likelihood of success than their counterparts. Some studies suggested that economic development should be deterministic to road safety performance because of the presence of a comprehensive road safety system management system, road infrastructure condition, level of motorization, road user behavior and education, and more importantly, the. technical and financial support devoted to the improvement of road infrastructures (ETSC, 2003; World Health Organization, 2004, Wegman et al., 2008, Bliss and Breen, 2009). Indeed, in the past decade, remarkable road fatality reductions were revealed in various countries across different clusters [Cluster 1: Portugal; Cluster 2: Ireland and Sweden; Cluster 3: Denmark, France, Iceland and Spain] regardless of the level of economic development and prevailing road safety risk. Also, countries in different clusters [Cluster 2: Norway and Sweden; Cluster 3: 
1 Denmark, Great Britain and Iceland] could have the same level of capability to maintain high 2 level of road safety management, and achieve sustainable safety gains (OECD, 2014). Therefore, 3 it should not be surprising that no evidence could be established for noticeable variation in the 4 likelihood of success across clusters that were categorized by prevailing road safety risk and economic development. Hence, no obvious association between cluster, target duration, target's

6 level of ambition, and target achievement could be revealed.

8 Indeed, collection and analysis of best road safety management practices in terms of policy developments, road safety strategies, and effective intervention have been the keys in monitoring the progress of road safety target. Some of the best practices are local traffic management scheme, automated enforcement, fatigue management programme, truck and bus warrant of fitness check, speed management, seat belt and helmet legislation, and alcohol ignition interlocks (Wegman et al., 2008; OECD, 2014). They are however not included in current quantitative analysis for the association between likelihood of target success and possible factors. Nevertheless, it would be worth investigating the effects of possible attributes in the planning of road safety policy and implementation of road safety action plan on the likelihood of target achievement in future qualitative research.

\subsection{Level of Ambition}

Our results indicate that an increase in the level of ambition is associated with a lower likelihood of target success. This is consistent with the findings of previous studies that although overly ambitious road safety targets, such as 'Vision Zero', may stimulate commitment from decision makers, they often prove to be spiritual substitutes that do not necessarily outperform their less ambitious counterparts. This addresses the importance of setting motivating yet rational action guidance and achievable targets (Rosencrantz et al., 2007; ETSC, 2011). Results of treatmentcomparison group before-after studies indicated that 3.5\% road fatality reduction per year, on average, could be revealed short-term after the target setting (Wong et al., 2006; Allsop et al., 2010). Therefore, targeted reduction rate at or above $4.5 \%$ could have been too ambitious.

One might argue that it is somewhat obvious that more ambitious the target, more difficult to realize. Indeed, quantified road safety targets have been set out based on different initiatives of respective countries including Vision Zero [Czech Republic, Denmark, Finland, Norway, Poland 
1 and Sweden], safe management system approach and sustainable safety [Australia, Austria, 2 Canada, Netherlands, New Zealand and Spain], and EU Road Safety Target and UN Road Safety 3 Plan for the Decade of Action of Road Safety [Argentina, Belgium and Malaysia] (OECD, 2014).

4 It is worth exploring the significance of the factors including type of road safety initiatives, 5 existence of continuous monitoring the progress of target achievement, setting up of high-level 6 outcome indicators, and implementation of specific target-driven safety program to the 7 likelihood of success (Kweon, 2010).

8

9 To determine the appropriate target's level of ambition, probabilities of success with respect to 10 different level of ambition of targets were estimated based on the coefficient estimates illustrated 11 in Model A of Table 4. Average values of vehicle ownership, target duration, and proportion of 12 completed target for each cluster were adopted. Values of target's level of ambition vary from $130 \%$ to $10 \%$. Figure 2 illustrates the estimated likelihoods of success and actual achievement, with 14 respect to different level of ambition, in different clusters.

[Insert Figure 2 here]

As illustrated in Figure 2, the likelihood of actual success (hollow circle) is seemingly higher, when the targeted fatality reduction rate is below $5 \%$. The estimated likelihood of success shows decreasing trend when the targeted annual reduction rate increases for all clusters. As Figure 2 shows, members of Cluster 1 (targets set by countries with low national income and high fatality rate) with targeted annual reduction rates below 6.4\%, members of Cluster 3 (targets set by countries of medium national income and low-medium fatality rate) with targeted annual reduction rates below $4.2 \%$, and members of Cluster 2 (targets set by countries of high national income and low-medium fatality rate) with targeted annual reduction rates below $3.4 \%$ respectively, may have $50 \%$ or higher likelihood of target success. Seemingly, it is appropriate for the countries with high level of economic development and low-medium fatality rate (Cluster 2) to set less ambitious targets than their counterparts.

\subsection{Target Duration}

We establish no significant association in this study between likelihood of success and duration of target. Yet, to determine the appropriate target duration, the probabilities of success with 
1 respect to different target duration were estimated based on the coefficient estimates illustrated in 2 Model A of Table 4. Average values of vehicle ownership, annual reduction rate, and proportion 3 of completed target for each cluster were adopted. Values of target duration vary from 3 to 18 4 years. Figure 3 illustrates the estimated likelihoods of success and actual achievement, with respect to different target duration, in different clusters.

$7 \quad$ [Insert Figure 3 here]

As illustrated in Figure 3, no obvious trend could be observed between the likelihood of actual success (hollow circle) and target duration. The likelihood of success is a trend that increases with target duration for all clusters. This finding is in line with the observation that a stronger trend in fatality reduction is achieved over a longer time, and the likelihood of success of such targets in the long run may be higher (Partyka, 1984).

As Figure 3 shows, members of Cluster 1 (targets set by countries of low national income and high fatality rate) have a consistently high likelihood of success (over 75\%) regardless of the target duration. On the other hand, members of Cluster 3 (targets set by countries of medium national income and low-medium fatality rate) with target duration longer than 16 years, and members of Cluster 2 (targets set by countries of high national income and low-medium fatality rate) with target duration longer than 11 years respectively, may have $50 \%$ or higher likelihood of target success. Seemingly, it is appropriate for the countries with moderate level of economic development and reasonably good prevailing safety performance (Cluster 3) to set longer term target.

Nevertheless, it is essential that periodic progress reviews be conducted and interim milestones related to the original target be set to achieve sustainable road safety goals (ETSC, 2003). For instance, experiences accumulated during the process should generate the input and effort required to ensure that the initial positive effect of target setting does not diminish (Wong and Sze, 2010). In addition, to ensure that the road safety strategies are successfully implemented, a wide range of action plans need to be introduced in different phases or entire duration of the target. For instances, various indicators including road crash, road fatality, traffic violation, and safety cost could be the base for the continuous monitoring of road safety development (OECD, 2014). Indeed, it might be essential to evaluate the intervention effects of the road safety policies 
1 that occur during the target period, rather than the characteristics of target itself, e.g. level of 2 ambition and duration, on the likelihood of success in future research. This could provide very 3 useful recommendations of future road safety action plans, based on the better understanding on

4 the relationship between target setting and revealed safety performance, and the reasons that 5 make the target setting work.

\section{CONCLUSION}

9 In this study, we diagnose 55 quantified road safety targets set in the period 1981 to 2009. The results of a cluster analysis indicate that the national income and fatality rate influence the patterns of targets. A binary logistic regression is applied to evaluate the likelihood of success of road safety targets and the factors that influence that success. The results indicate that targets with lower level of ambition have a higher likelihood of success than those of the counterparts. This reinforces the finding of previous studies that setting rational and yet achievable quantified road safety targets is essential. The level of ambition should vary with the level of economic development and fatality rate. Yet, no evidence could be established for the relationship between national income, fatality rate, target duration and likelihood of success. This suggests that the emphasis on road safety management strategies and programs should differ across countries, regardless of the economic performance and road safety risk. It would be worth investigating cost-effective strategies and measures that could help improve the likelihood of success with an eye to achieving sustainable road safety improvements in future qualitative research.

\section{ACKNOWLEDGEMENTS}

The work that is described in this paper was supported by research grants from the University

Research Committee (Project Nos. 201109176069) of the University of Hong Kong, and a grant from the Research Grants Council of the Hong Kong Special Administrative Region, China (Project No. HKU7175/12E).

\section{REFERENCES}

Anderson, T.K. Kernel density estimation and K-means clustering to profile road accident hotspots. Accident Analysis and Prevention, Vol. 41, 2009, pp. 359-364. 
1 Allsop, R.E., N.N. Sze, and S.C. Wong. A note on the association between setting quantified

road safety targets and road fatality reduction. Accident Analysis and Prevention, Vol. 43, 2011, pp. 1279-1283.

Bliss, T., and J. Breen. Implementing the Recommendations of the World Report on Road Traffic Injury Prevention. World Bank Global Road Safety Facility. Washington DC, United States, 2009.

World Health Organization. World Report on Traffic Injury Prevention. World Health Organization, Geneva, 2004.

Depaire, B., G. Wets, and K. Vanhoof. Traffic accident segmentation by means of latent class clustering. Accident Analysis and Prevention, Vol. 40, 2008, pp. 1257-1266.

Elvik, R. Quantified road safety targets: a useful tool for policy-making? Accident Analysis and Prevention, Vol. 25, 1993, pp. 569-583.

Elvik, R. Quantified Road Safety Targets: An Assessment of Evaluation Methodology. ТØI Report 539/2001, Institute of Transport Economics, Oslo, 2001.

Elvik, R. Road safety management by objectives: a critical analysis of the Norwegian approach. Accident Analysis and Prevention, Vol. 40, 2008, pp. 1115-1122.

European Transport Safety Council (ETSC). Assessing Risk and Setting Targets in Transport Safety Programmes. ETSC, Brussels, 2003.

European Transport Safety Council (ETSC). 2010 Road Safety Target Outcome: 100,000 Fewer Deaths Since 2001, $5^{\text {th }}$ Road Safety PIN Report. ETSC, Brussels, 2011.

Hauer, E. (1997) Observational Before-After Studies in Road Safety. Elsevier Science Ltd., Oxford, UK.

Hosmer, D.W., and S. Lemeshow. Applied Logistic Regression. Wiley-Interscience, New York, 2000.

International Road Federation (IRF). Annual Report of IRF World Road Statistics. IRF, Geneva, 2013.

Kweon, Y.-J. Data-driven reduction targets for a highway safety plan. Transport Policy, Vol. 17, 2010, pp. 230-239.

OECD Scientific Expert Group. Targeted Road Safety Programmes. OECD, Paris, 1994.

OECD Scientific Expert Group. Towards Zero: Ambitious Road Safety Targets and the Safe System Approach. OECD, Paris, 2008.

OECD Scientific Expert Group. IRTAD Annual Report 2011. OECD, Paris, 2012.

OECD Scientific Expert Group. IRTAD Annual Report 2014. OECD, Paris, 2014. 
1 R Core Team. R: A Language and Environment for Statistical Computing. R Foundation for Statistical Computing, Vienna, Austria, 2013.

Shen, Y., E. Hermans, T. Brijs, G. Wets, and K. Vanhoof. Road safety risk evaluation and target setting using data envelopment analysis and its extensions. Accident Analysis and Prevention, Vol. 48, 2012, pp. 430-441.

Tingvall, C., H. Stigson, L. Eriksson, R. Johansson, M. Krafft, and A. Lie. The properties of

Appendix: Study Reports and Technical Papers on National Quantified Road Safety

\section{Targets}

Austrian Road Safety Fund. Austrian Road Safety Programme 2011-2020. Austrian Ministry for Transport, Innovation and Technology, Vienna, 2011.

http://www.bmvit.gv.at/en/service/publications/downloads/rsp2020.pdf. Accessed 13 June 2013. Australian Transport Council. National Road Safety Strategy 2011-2020. Australian Transport Council, Australia, 2011.

http://www.atcouncil.gov.au/documents/files/NRSS_2011_2020_15Aug11.pdf. Accessed 13 June 2013. 
1 Canadian Council of Motor Transport Administrators. Annual Report of Road Safety Vision 2010, 2 Transport Canada, Canada, 2005.

3 http://www.ccmta.ca/english/committees/rsrp/rsv/rsv.cfm. Accessed 13 June 2013.

4 Canadian Council of Motor Transport Administrators. Canada's Road Safety Strategy 2015. 5 Transport Canada, Canada, 2011.

6 http://www.ccmta.ca/crss-2015/_files/road_safety_strategy_2015.pdf. Accessed 13 June 2013.

7 Department of Transport, Ireland. Road Safety Strategy 2007-2012. Road Safety Authority, 8 Ireland, 2007.

9 http://www.rsa.ie/Documents/Road\%20Safety/RSA_Strategy_ENG_s.pdf. Accessed 13 June 102013.

11 European Transport Safety Council (ETSC). A Methodological Approach to National Road 12 Safety Policies. ETSC, Brussels, 2006.

13 http://www.etsc.eu/documents/A_methodological_approach_to_national_road_safety_policies.p 14 df. Assessed 13 June 2013.

15 European Transport Safety Council (ETSC). Assessing Risk and Setting Targets in Transport 16 Safety Programmes. ETSC, Brussels, 2006.

17 http://www.etsc.eu/documents/riskassess.pdf. Accessed 13 June 2013.

18 Kannellaidis, G., G. Yannis, S. Vardaki, A. Laiou, and C. Voulgari, C. Development of a Strategic

19 Plan for the Improvement of Road Safety in Greece, 2011-2020. National Technical University 20 of Athens, Athens, 2011.

21 http://nrso.ntua.gr/geyannis/images/stories/ge/Research/ntua-rss3-rn54sen.pdf. Accessed 13 June 22 2013.

Ministry of Transport, New Zealand. Road Safety to 2010. Ministry of Transport, New Zealand, 2002.

http://www.transport.govt.nz/ourwork/Documents/2010-strategy.pdf. Accessed 28 November 2012.

Ministry of Transport and Communication, Finland. Road Safety 2006-2010. Ministry of 28 Transport and Communication, Finland, 2006.

29 http://www.lvm.fi/fileserver/road\%20safety\%202006-2010.pdf. Accessed 13 June 2013.

30 OECD Scientific Expert Group. IRTAD Annual Report 2011. OECD, Paris, 2012.

31 http://internationaltransportforum.org/irtadpublic/pdf/11IrtadReport.pdf. Accessed 13 June 2013

32 OECD Scientific Expert Group. IRTAD Annual Report 2014. OECD, Paris, 2014.

33 http://internationaltransportforum.org/irtadpublic/pdf/14IrtadReport.pdf. Accessed 1 July 2014. 
1 Swedish Transport Administration. Review of Interim Targets and Indicators for Road Safety in 2 2010-2020 - Analytic Report. Swedish Transport Administration, Sweden, 2012.

3 http://publikationswebbutik.vv.se/upload/6816/2012_162_review_of_interim_targets_and_indica 4 tors_for_road_safety_in_2010_2020.pdf. Accessed 13 June 2013.

5 Transport Canada. Canada's Road Safety Targets to 2010. Minister of Public Works and 6 Government Services, Canada, 2001.

7 http://www.ccmta.ca/english/pdf/rsv_report_2010targets_e.pdf. Accessed 13 June 2013.

8 World Health Organization. World Report on Road Traffic Injury Prevention. Wealth Health 9 Organization, Geneva, 2004.

10 http://www.who.int/violence_injury_prevention/publications/road_traffic/world_report/en/index. 11 html. Accessed 13 June 2013.

12 Yannie, G., and E. Papadimitriou. Road safety in Greece. Paper presentation at the Transport 13 Research Arena Europe 2012, Athens, 23-26 April 2012.

14 http://nrso.ntua.gr/geyannis/images/stories/ge/Publications/Presentations-conferences/geyannis15 pc131c.pdf. Accessed 13 June 2013 


\section{TABLES AND FIGURES}

2

3 Table 1. Details of Targets under Investigation

\begin{tabular}{|c|c|c|c|c|c|c|c|}
\hline Country & Base Year & Target Year & Target* & Country & Base Year & Target Year & Target* \\
\hline Argentina & 2009 & 2014 & $50 \%$ & Israel & 2005 & 2010 & $24 \%$ \\
\hline Australia & 1992 & 2001 & $2 \% \wedge$ & Italy & 2001 & 2010 & $50 \%$ \\
\hline Australia & 1997 & 2005 & $10 \%$ & Japan & 2000 & 2010 & $14 \%{ }^{\wedge}$ \\
\hline Australia & 2001 & 2010 & $40 \%$ & Korea & 2007 & 2012 & $50 \%$ \\
\hline Austria & $1998-2000$ & 2004 & $25 \%$ & Lithuania & 2004 & 2010 & $50 \%^{\wedge}$ \\
\hline Austria & $1998-2000$ & 2010 & $50 \%$ & Malaysia & 2005 & 2010 & $27 \%$ \\
\hline Belgium & 2001 & 2006 & $28 \%$ & Netherlands & 1985 & 2000 & $25 \%$ \\
\hline Belgium & 2007 & 2010 & $30 \%$ & Netherlands & 1998 & 2010 & $30 \% \wedge$ \\
\hline Canada & 1996-2001 & 2008-2010 & $30 \%$ & Netherlands & 2008 & 2020 & $33 \% \wedge$ \\
\hline Czech Republic & 2002 & 2010 & $50 \%^{\wedge}$ & New Zealand & 1990 & 1994 & $11 \%{ }^{\wedge}$ \\
\hline Denmark & 1986-1988 & 2000 & $40 \%$ & New Zealand & 1990 & 2001 & $42 \%$ \\
\hline Denmark & 1998 & 2012 & $40 \%^{\wedge}$ & New Zealand & 1999 & 2010 & $42 \%$ \\
\hline Denmark & 2006 & 2012 & $35 \%^{\wedge}$ & Norway & 1984-1986 & 1993 & $0 \%^{\wedge}$ \\
\hline Finland & 1986 & 1994 & $40 \%$ & Norway & 2004 & 2015 & $30 \% \wedge$ \\
\hline Finland & 1988 & 2000 & $50 \%$ & Norway & 2009 & 2020 & $33 \%^{\wedge}$ \\
\hline Finland & 2000 & 2010 & $37 \%$ & Poland & 1991 & 2001 & $24 \% \wedge$ \\
\hline France & 1997 & 2002 & $50 \%$ & Poland & 1997-1999 & 2010 & $43 \%^{\wedge}$ \\
\hline France & 2007 & 2012 & $35 \%$ & Poland & 2003 & 2013 & $50 \%$ \\
\hline Great Britain & 1981-1985 & 2000 & $33 \%^{\wedge}$ & Portugal & $1998-2000$ & 2009 & $50 \%^{\wedge}$ \\
\hline Great Britain & 1994-1998 & 2010 & $40 \%^{\wedge}$ & Portugal & 2009 & 2011 & $1 \%$ \\
\hline Great Britain & 2005-2009 & 2020 & $37 \%^{\wedge}$ & Spain & 1992 & 1999 & $30 \%$ \\
\hline Greece & 2000 & 2005 & $20 \%$ & Spain & 2003 & 2008 & $40 \% \wedge$ \\
\hline Greece & 2000 & 2015 & $40 \%^{\wedge}$ & Sweden & 1989 & 2000 & $56 \%$ \\
\hline Hungary & 2001 & 2010 & $30 \%^{\wedge}$ & Sweden & 1996 & 2007 & $50 \%$ \\
\hline Iceland & 1991-1996 & 2000 & $11 \%$ & Sweden & 2007 & 2020 & $50 \%^{\wedge}$ \\
\hline Iceland & 1999-2003 & 2016 & $50 \% \wedge$ & Switzerland & 2000 & 2010 & $50 \%$ \\
\hline Ireland & 1997 & 2002 & $20 \% \wedge$ & United States & 1996 & 2008 & $20 \%$ \\
\hline Ireland & 2007 & 2012 & $20 \% \wedge$ & & & & \\
\hline
\end{tabular}

4 * Percentage reduction in road fatalities in the target year compared to the base year

$5 \wedge$ Target that is regarded as being achieved

6 
1 Table 2. Summary Statistics of Target under Investigation

\begin{tabular}{lcccc}
\hline Factor & $\begin{array}{c}\text { Mean/ } \\
\text { Proportion }\end{array}$ & $\begin{array}{c}\text { Standard } \\
\text { Deviation }\end{array}$ & Minimum & Maximum \\
\hline Fatality Rate (per 100,000 inhabitant) & 5.84 & 3.48 & 2.8 & 23.6 \\
Vehicle Ownership (per 1000 inhabitant) & 639.63 & 108.21 & 344 & 846 \\
GDP per capita (,000 USD) & 36.04 & 9.47 & 17.2 & 55.9 \\
Target Duration (year) & 9.05 & 3.57 & 2 & 17 \\
Annual Average Reduction Rate & 0.05 & 0.03 & 0 & 0.13 \\
Completed Target & 0.84 & 0.37 & 0 & 1 \\
\hline
\end{tabular}

2 
1 Table 3. Preliminary Analysis for Possible Factors Influencing Target Success

\begin{tabular}{lcccc}
\hline Factor & Total & Success $(\%)$ & Fail $(\%)$ & $\chi^{2}(p$-level $)$ \\
\hline $\begin{array}{l}\text { Vehicle Ownership } \\
\quad \text { 650 per 1000 inhabitant }\end{array}$ & 27 & $15(55.6 \%)$ & $12(44.4 \%)$ & $2.18(0.14)^{\wedge}$ \\
$\quad>$ =650 per 1000 inhabitant & 28 & $10(35.7 \%)$ & $18(64.3 \%)$ & \\
$\begin{array}{l}\text { Target Duration } \\
\quad \text { Less than 10 years }\end{array}$ & 28 & $11(39.3 \%)$ & $17(60.7 \%)$ & \\
$\quad$ Greater than/equal to 10 years & 27 & $14(51.9 \%)$ & $13(48.1 \%)$ & \\
Level of ambition & & & & $11.55(<0.01)^{* *}$ \\
$\quad$ ARR < 4.5\% & 28 & $19(67.9 \%)$ & $9(32.1 \%)$ & \\
$\quad$ ARR >= 4.5\% & 27 & $6(22.2 \%)$ & $21(77.8 \%)$ & \\
Target Type & & & & $4.53(0.03)^{*}$ \\
$\quad$ In-Progress & 9 & $7(77.8 \%)$ & $2(22.2 \%)$ & \\
$\quad$ Completed & 46 & $18(39.1 \%)$ & $28(60.9 \%)$ & \\
\hline
\end{tabular}

$2 \wedge$ At the $15 \%$ level of significance.

$3 *$ At the 5\% level of significance.

$4 \quad * *$ At the $1 \%$ level of significance. 
1 Table 4. Consolidated Analysis of Possible Factors Influencing Target Success

\begin{tabular}{|c|c|c|c|c|}
\hline \multirow[t]{2}{*}{ Factor } & \multicolumn{2}{|c|}{ Model A } & \multicolumn{2}{|c|}{ Model B } \\
\hline & Coeff. (S.E.) & z-Value (p-level) & Coeff. (S.E.) & z-Value (p-level) \\
\hline Constant & $5.15(2.83)$ & $1.82(0.07)^{\wedge}$ & $2.91(1.37)$ & $2.13(0.03)^{*}$ \\
\hline Cluster 1 & $0.64(0.91)$ & $0.70(0.49)$ & $0.19(0.92)$ & $0.21(0.84)$ \\
\hline Cluster 2 & \multicolumn{4}{|c|}{ (Control) } \\
\hline Cluster 3 & $0.39(0.74)$ & $0.52(0.60)$ & $0.38(0.78)$ & $0.49(0.63)$ \\
\hline Vehicle Ownership ${ }^{\text {(Note) }}$ & $-0.005(0.003)$ & $-1.76(0.08)^{\wedge}$ & \multicolumn{2}{|c|}{-} \\
\hline Vehicle Ownership <650 (Note) & & & \multicolumn{2}{|c|}{ (Control) } \\
\hline Vehicle Ownership $>=650^{\text {(Note) }}$ & & . & $-0.92(0.69)$ & $-1.33(0.18)$ \\
\hline Target Duration & $0.07(0.10)$ & $0.65(0.52)$ & \multicolumn{2}{|c|}{-} \\
\hline Target Duration $<10$ years & & . & \multicolumn{2}{|c|}{ (Control) } \\
\hline Target Duration $>=10$ years & & . & $-0.56(0.75)$ & $-0.75(0.46)$ \\
\hline Level of ambition (ARR) & $-26.84(13.31)$ & $-2.02(0.04)^{*}$ & \multicolumn{2}{|c|}{-} \\
\hline $\mathrm{ARR}<4.5 \%$ & & - & \multicolumn{2}{|c|}{ (Control) } \\
\hline $\mathrm{ARR}>=4.5 \%$ & & . & $-2.19(0.72)$ & $-3.06(0.002)^{* *}$ \\
\hline In-Progress & \multicolumn{4}{|c|}{ (Control) } \\
\hline Completed & $-1.74(1.00)$ & $-1.75(0.08)^{\wedge}$ & $-1.89(1.03)$ & $-1.84(0.07)^{\wedge}$ \\
\hline \multicolumn{5}{|l|}{ Model Performance } \\
\hline Number of observations & 55 & & 55 & \\
\hline AIC & 75.404 & & 71.804 & \\
\hline
\end{tabular}

2 Note: per 1000 inhabitants

$3 \wedge$ At the 10\% level of significance.

$4 *$ At the $5 \%$ level of significance.

$5 \quad * *$ At the $1 \%$ level of significance.

6 
1 Table 5. Analysis of Interaction Effects on the Likelihood of Target Success

\begin{tabular}{lcc}
\hline Factor & Coeff. (S.E.) & z-Value (p-level) \\
\hline Constant & $3.73(1.66)$ & $2.24(0.03)^{*}$ \\
Cluster & & \\
$\quad$ Cluster 1 & $-1.60(1.52)$ & $-1.05(0.29)$ \\
$\quad$ Cluster 2 & (Control) & \\
$\quad$ Cluster 3 & $0.22(1.52)$ & $0.15(0.88)$
\end{tabular}

Vehicle Ownership

Vehicle Ownership $<650^{\text {(Note) }}$

(Control)

Vehicle Ownership $>=650^{\text {(Note) }}$

$-1.00(0.75)$

$-1.34(0.18)$

Target Duration

Target Duration $<10$ years

(Control)

Target Duration $>=10$ years

$-1.21(0.23)$

Level of Ambition (ARR)
$\mathrm{ARR}<4.5 \%$
(Control)
$\mathrm{ARR}>=4.5 \%$
$-2.41(1.34)$
$-1.80(0.07)^{\wedge}$

Target Type

In-Progress

(Control)

Completed

$-2.03(1.11)$

$-1.82(0.07)^{\wedge}$

\begin{tabular}{lll}
\hline Interaction Effect & \\
\hline Cluster $1 \times$ Duration $>=10$ years & $3.27(2.07)$ & $1.59(0.11)$ \\
Cluster $3 \times$ Duration $>=10$ years & $0.33(1.81)$ & $0.18(0.86)$ \\
Cluster $1 \times$ ARR $>=4.5 \%$ & $0.48(2.11)$ & $0.23(0.82)$ \\
Cluster $3 \times$ ARR $>=4.5 \%$ & $-0.24(1.87)$ & $-0.13(0.90)$
\end{tabular}

Model Performance

Number of observations

55

AIC

76.329

2 Note: per 1000 inhabitants

$3 \wedge$ At the $10 \%$ level of significance.

$4 *$ At the $5 \%$ level of significance.

5 
1 Figure 1. Dendrogram of the Cluster Analysis of Quantified Road Safety Targets

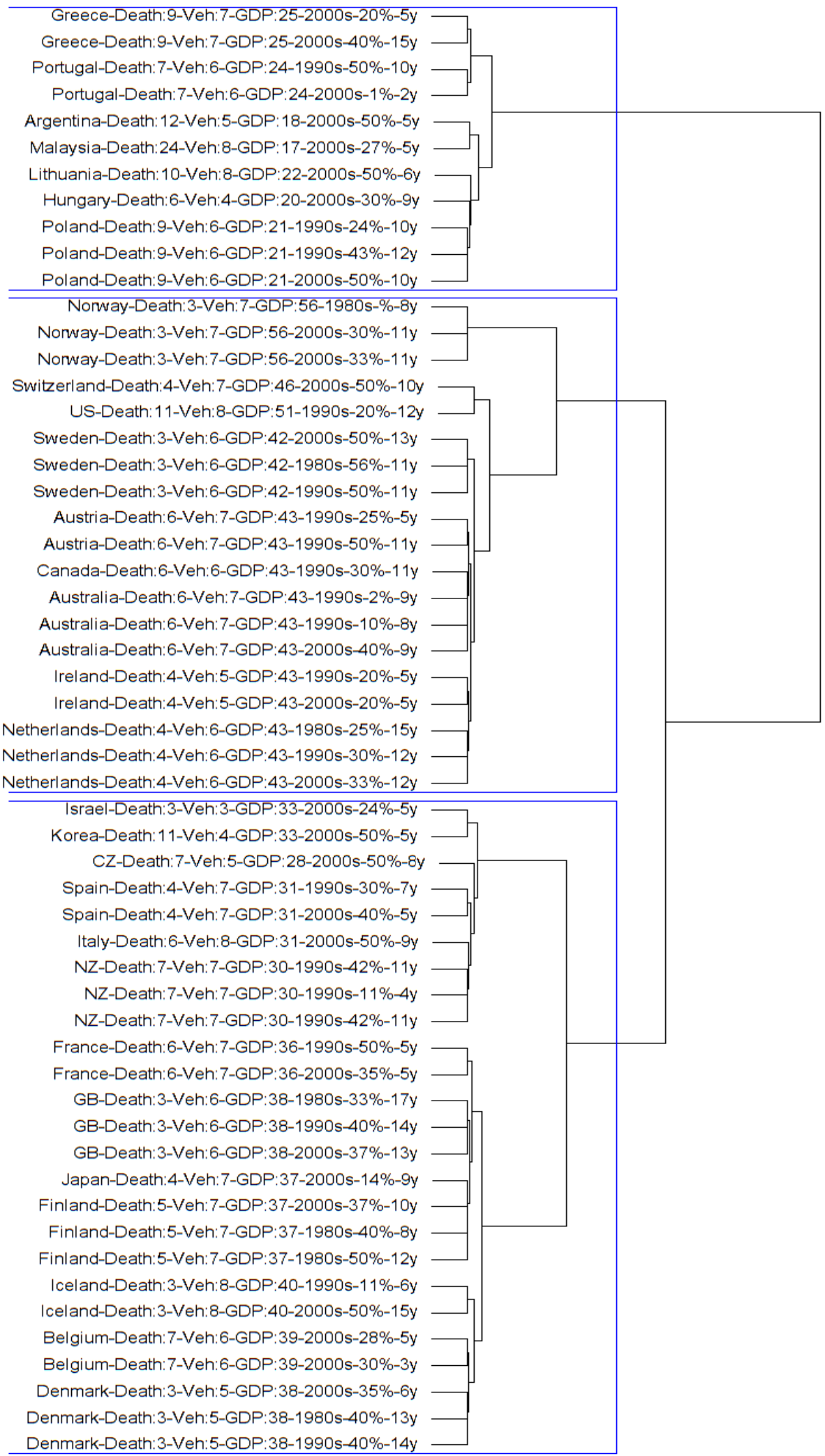

3 Notes:

4 Death - Road fatality per 100,000 inhabitant; Veh - car per 100,000 inhabitant; GDP - 000 5 USD per capita 
1 Figure 2. Relationships between a Target's Likelihood of Success and Level of Ambition

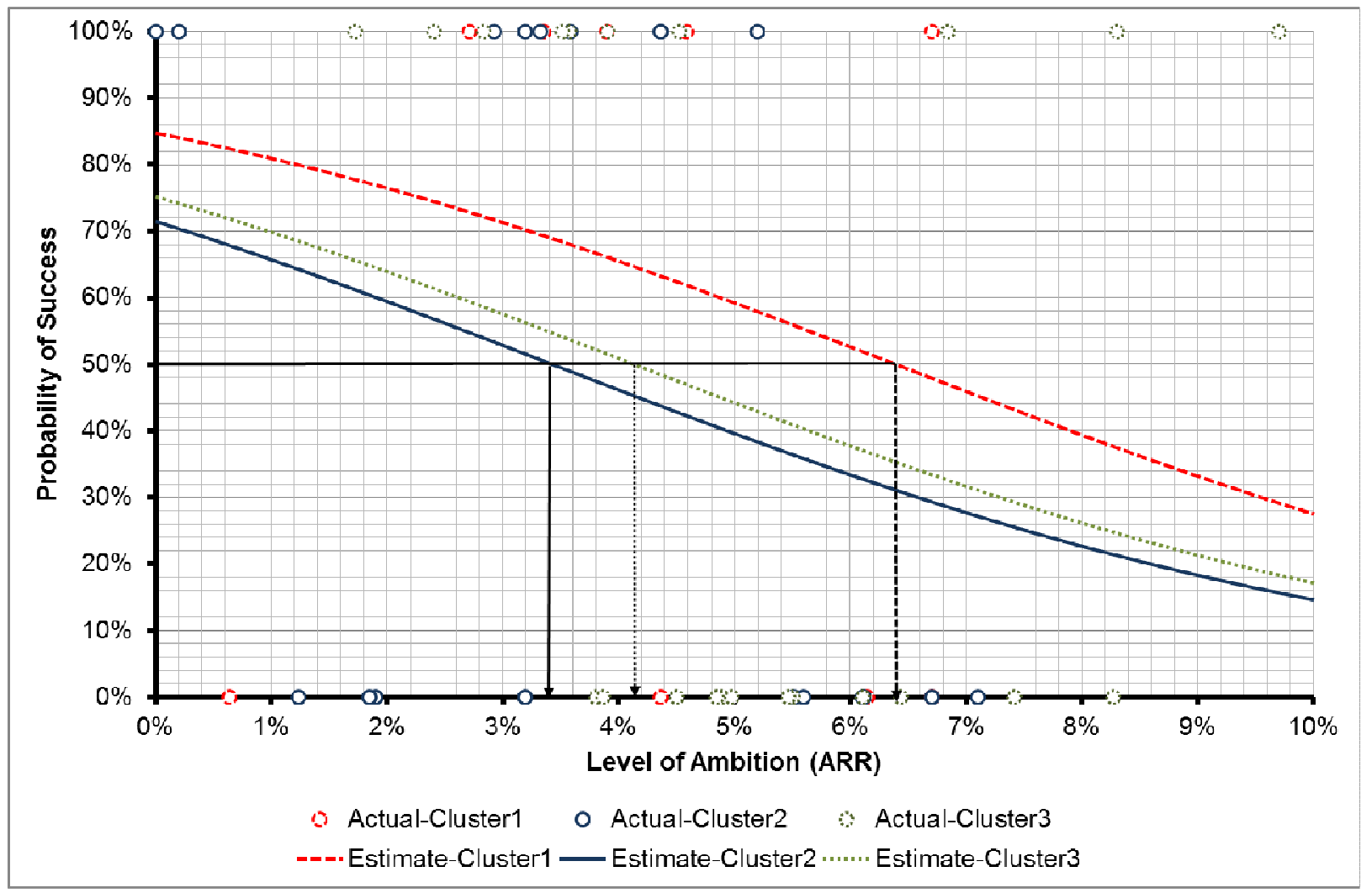

3 
1 Figure 3. Relationships between a Target's Likelihood of Success and Target Duration

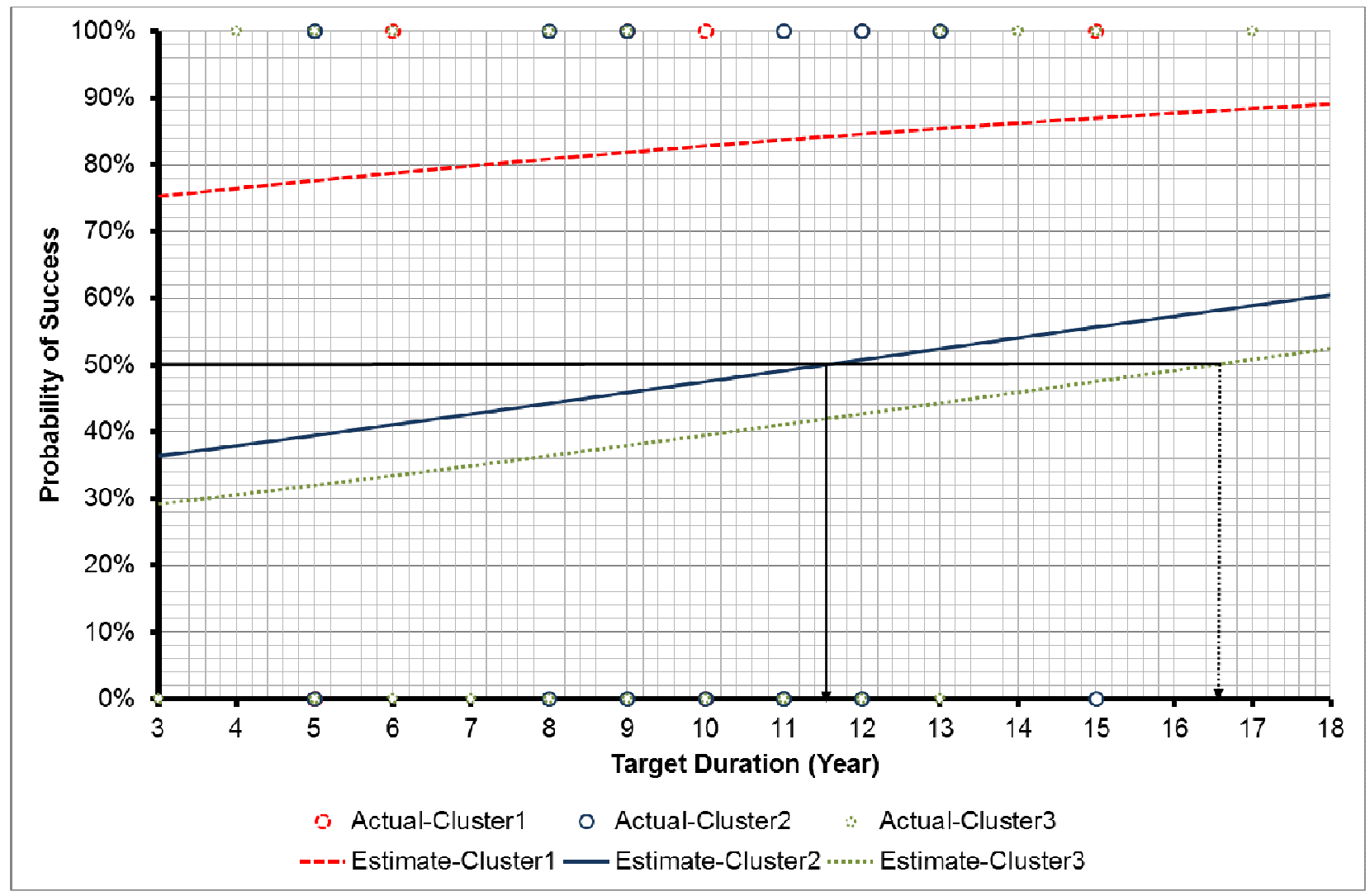

